\title{
RESILIENCIA COMO PREDICTORA DE DEPRESIÓN EN MUJERES CON CÁNCER DE MAMA
}

\author{
Paula Ruiz-González \\ Laboratorio de Inteligencia Emocional \\ Universidad de Cádiz (Cádiz, España) \\ Instituto de Investigación e Innovación \\ Biomédica de Cádiz (INIBICA) (Cádiz, España) \\ paula.ruiz@uca.es \\ Antonio Zayas \\ Laboratorio de Inteligencia Emocional \\ Universidad de Cádiz (Cádiz, España) \\ Lucía Morales-Sánchez \\ Laboratorio de Inteligencia Emocional \\ Universidad de Cádiz (Cádiz, España) \\ Paloma Gil-Olarte \\ Laboratorio de Inteligencia Emocional \\ Universidad de Cádiz (Cádiz, España) \\ Rocío Guil \\ Laboratorio de Inteligencia Emocional \\ Universidad de Cádiz (Cádiz, España)
}

Fecha de Recepción: 2 Febrero2 019

Fecha de Admisión: 30 Abril 2019

\section{RESUMEN}

El diagnóstico de cáncer de mama supone, en ocasiones, la vivencia de un acontecimiento traumático que incrementa la vulnerabilidad a padecer trastornos mentales como la depresión. Sin embargo y a pesar del sufrimiento, las mujeres se ven más fortalecidas y resilientes tras la superación de la enfermedad.

El propósito de este estudio fue: 1) Explorar los niveles de depresión y resiliencia en mujeres supervivientes de cáncer de mama y sin cáncer, y 2) Analizar la capacidad explicativa y predictiva de la resiliencia sobre la depresión, controlando el efecto del diagnóstico de cáncer y la edad.

La muestra estuvo conformada por 110 mujeres (40.9\% supervivientes de cáncer de mama, $50.9 \%$ sin cáncer) cuya media de edad fue 44.27 (DT= 11.79; rango = 25-69). Se administró la Escala de Ansiedad y Depresión Hospitalaria -HADS-57- (Zigmond y Snaith, 1983; adaptación al castellano de Rico, Restrepo y Molina, 2006) y la Escala de Resiliencia (Wagnild y Young, 1993; adaptación al castellano de Novella, 2002). 


\section{RESILIENCIA COMO PREDICTORA DE DEPRESIÓN EN MUJERES CON CÁNCER DE MAMA}

Se realizó un análisis de varianza que reveló como las supervivientes mostraban, de forma estadísticamente significativa, mayores niveles de resiliencia $(F[1,108]=9.82 ; p<.01)$, no así en depresión $(F[1,109]=2.81, p>.05)$. Un análisis de regresión múltiple por pasos indicó que la resiliencia explicó el $28 \%$ de la varianza de la depresión $\left(\mathrm{R}^{2}=.28\right)$, siendo su capacidad predictiva negativa y estadísticamente significativa ( $\beta=-.09$ ). Dicha capacidad explicativa se vio incrementada significativamente en el modelo $2\left(\mathrm{R}^{2}=.31\right)$ por el efecto de haber sido diagnosticada de cáncer.

Estos datos aportan apoyo empírico a la necesidad de abordar los problemas que conducen a una depresión en supervivientes de cáncer de mama. El refuerzo de la resiliencia como factor de protección, amortiguará el impacto del cáncer sobre el desarrollo de sintomatología depresiva, facilitando la adaptación y la prevención de trastornos emocionales una vez superada la enfermedad.

Palabras clave: depresión; resiliencia; cáncer de mama; mujeres

\section{ABSTRACT}

Resilience as a predictor of depression in women with breast cancer. Breast cancer diagnosis involves, in many cases, a traumatic event experience that increases vulnerability to suffer mental disorders such depression. However, despite the suffering, women are more strengthened and resilient after overcoming with disease.

The purpose of this study was: 1) To explore depression and resilience in women survivors of breast cancer survivors and women without cancer, and 2) To analyze the explanatory and predictive ability of resilience on depression, controlling for the effect of cancer diagnosis and women's age.

The sample consisted of 110 women (40.9\% breast cancer survivors, $50.9 \%$ women without cancer) whose average age was $44.27(S D=11.79$; range= 22-69). The Hospital Anxiety and Depression Scale -HADS-57- (Zigmond \& Snaith, 1983; Spanish versión by Rico, Restrepo \& Molina, 2006) and the Resilience Scale (Wagnild \& Young, 1993; Spanish versión by Novella, 2002) were administered.

The analysis of the variance revealed how survivors showed, in a statistically significant way, higher levels of resilience $(F[1,108]=9.82 ; p<.01)$, but not in depression $(F[1,108]=2.81 ; p>.05)$. A stepwise multiple regression analysis indicated that resilience explained $28 \%$ of the variance in depression $\left(R^{2}=.28\right)$, being its predictive ability negative and statistically significant $(\beta=-.09)$. This explanatory capacity was increased significantly in model $2\left(R^{2}=.31\right)$ due to the effect of having been diagnosed with cancer.

These data provide empirical support concerning the need to address problems that lead to depression in breast cancer survivors. Specifically, enhacing resilience as a protective factor will cushion the impact of cancer on the development of depressive symptomatology, facilitating the adaptation and prevention of emotional disorders once the disease has been overcome.

Keywords: depression; resilience; breast cancer; women

\section{ANTECEDENTES}

El cáncer es una de las enfermedades más significativas a nivel mundial por su elevada incidencia y morbilidad (OMS, 2017). En 2017 los nuevos casos de cáncer en España han sido 228.482, estimándose 315.413 nuevos casos para el 2035 (Sociedad Española de Oncología Médica, 2018).

Respecto al cáncer de mama, representa el 16\% de todos los cánceres femeninos (Agencia Internacional para la Investigación sobre Cáncer de la OMS, 2002; Sociedad Española de Oncología Médica, 2017). En Andalucía son diagnosticados cada año alrededor de 4.900 nuevos casos, no obstante, la supervivencia ha aumentado considerablemente en los últimos 25 años, situándose 
actualmente en un 85.6\%, según los últimos datos del Registro de Cáncer Andaluz. Concretamente, Cádiz es una de las provincias que, a pesar de tener un patrón de mortalidad descendente, presenta una mayor mortalidad relativa tanto si se compara con el conjunto de Andalucía, como con España en general (22.08 defunciones por cada 100.000 habitantes en el periodo constatado 19882000).

Según la OMS (2004), la enfermedad física, especialmente de carácter crónico como el cáncer, supone un factor de riesgo ante el desarrollo de problemas de salud mental como la depresión (Acosta et al., 2017). El diagnóstico de cáncer de mama suele ser una experiencia traumática que genera reacciones emocionales adversas como ansiedad, depresión, tristeza y miedo, alterando el estado psicológico de las pacientes y afectando a múltiples esferas de su vida diaria (Molano y Varela, 2017).

Krebber et al. (2014) señalan la frecuente comorbilidad entre el cáncer de mama y distintas alteraciones emocionales como limitadoras para el afrontamiento de la enfermedad. Específicamente, se han encontrado tasas de depresión que oscilan entre el 15-58\% en pacientes de cáncer de mama, tanto sometidas a tratamiento como en supervivientes de la enfermedad (Montiel et al., 2016; Vázquez et al., 2015).

Además, se ha encontrado que la prevalencia de depresión en mujeres con cáncer es el doble que en mujeres sin cáncer, siendo especialmente mayor durante el primer año tras recibir el diagnóstico (Burgess et al., 2005; Valderrama y Sánchez, 2018) e incluso una vez superada la enfermedad (Mitchell, Ferguson, Gill, Paul y Symonds, 2014). Sin embargo, otros estudios predicen bajas tasas de depresión y buena calidad de vida en supervivientes de cáncer (Baker, Denniston, Haffer y Liberatos, 2009). A pesar de la gran cantidad de estudios que relacionan al cáncer con la depresión, en muchas ocasiones su diagnóstico pasa desapercibido debido a la confluencia de sintomatología común al proceso oncológico, tales la fatiga, el cansancio, la pérdida de apetito 0 alteraciones del sueño (Newport y Nemeroff, 1998).

Según García-Herrera, Nogueras, Muñoz y Morales (2011) la depresión se caracteriza por ausencia de afectividad positiva, tristeza, pérdida del interés y de la capacidad de disfrute, bajo estado de ánimo y presencia de síntomas emocionales, cognitivos, físicos y conductuales. Además del malestar que provoca, la presencia de este trastorno parece afectar a la evolución clínica de las pacientes, disminuyendo la adherencia al tratamiento, prolongado las visitas médicas e ingresos hospitalarios y reduciendo las posibilidades de supervivencia, lo que indudablemente tiene repercusiones sobre su calidad de vida (Rodríguez, Amboage, Blázquez, Torres y Gaviria, 2015).

Sin embargo, se ha encontrado que el ser humano puede hacer uso de recursos personales que actúan como factores protectores ante el padecimiento de este tipo de alteraciones psicológicas y emocionales, como es el caso de la resiliencia. Este concepto hace referencia a la capacidad de generar respuestas asociadas al crecimiento y maduración personal, así como la aceptación de adversidades otorgándoles significado positivo (Guil et al., 2016).

García, López y Latorre (2015), encuentran mayor capacidad de resistir y tolerar acontecimientos adversos en mujeres que padecen cáncer de mama en comparación con un grupo de mujeres sanas. Además, esta capacidad parece ser mayor especialmente en mujeres que perciben beneficios derivados del padecimiento y tratamiento de la enfermedad (García-Serrablo, Acevedo y Ortiz, 2011). También se ha encontrado mayor crecimiento personal en aquellas que han sobrevivido al cáncer, al haber sido capaces de manejar sentimientos y preocupaciones derivados del futuro incierto que les subyace (Fernández y García, 2012).

Los hallazgos empíricos concluyen un mayor ajuste y bienestar psicológico en mujeres con cáncer de mama tras encontrar sentido a experiencias traumáticas (Bellizzi y Blank, 2006). Además, se 
ha observado que las cogniciones positivas contrarrestan el impacto de afectos negativos y estrés asociados al cáncer (Cucarella, 2013). Así, se establece a la resiliencia como predictor negativo de la depresión, mostrando que las pacientes con elevada resiliencia manifiestan menos secuelas emocionales y síntomas depresivos (Gotay, Ransom y Pagano, 2007; Hu, Xiao, Peng, Kuang y He, 2018; Markovitz, Schrooten, Arntz y Peters, 2015; Valderrama y Sánchez, 2018).

El interés de este estudio radica en hacer frente a la controversia acerca de la existencia de depresión en pacientes de oncología mamaria. La muestra de estudio será superviviente de la enfermedad, entendiendo que algunos problemas psicológicos y emocionales persisten a pesar de haberse superado el cáncer, lo que indudablemente afecta a su salud y calidad de vida. Además, es especialmente relevante abordar aquellas variables psicológicas que predicen la aparición de sintomatología depresiva para una detección temprana del trastorno, permitiendo ser la base sobre la que ofrecer una óptima atención psicológica que contribuya a mejorar el bienestar psicológico de las mujeres (Viniegras y Blanco, 2010).

\section{OBJETIVOS}

Conocer los niveles de depresión y resiliencia en un grupo de mujeres supervivientes de cáncer de mama y sin cáncer.

Observar si existen diferencias significativas en depresión y resiliencia entre ambos grupos.

Analizar la capacidad explicativa y/o predictiva de la resiliencia sobre la depresión controlando el efecto de haber sido diagnosticada de cáncer y la edad de las mujeres.

\section{PARTICIPANTES}

La muestra estuvo conformada por 110 mujeres de la provincia de Cádiz con edades comprendidas entre los 25 y 69 años ( $M=44.27 ; D T=11.79)$. El 40.9\% eran supervivientes de cáncer de mama y el $59.1 \%$ no padecían ningún tipo de cáncer.

Los criterios de inclusión comunes a ambos grupos fueron: 1) Ser mujer, 2) Tener más de 18 años, 3) Tener un nivel de lectura y escritura que posibilitara la comprensión de las escalas de evaluación, 4) No encontrarse, en el momento del estudio en tratamiento psiquiátrico y/o psicológico debido a un trastorno mental grave y 5) No estar en el momento de la entrevista, ni en los últimos tres meses, bajo medicación psicoactiva. Además de los indicados anteriormente, para el grupo de mujeres supervivientes de cáncer de mama se tuvo en cuenta: 1) Haber recibido un diagnóstico de cáncer de mama al menos un año antes de participar en el estudio o haber recibido el alta médica.

Las mujeres que cumplían los criterios de inclusión y, que de forma voluntaria accedieron a participar, fueron citadas de forma grupal y recibieron un consentimiento informado donde se comunicaba la voluntariedad del estudio, el carácter anónimo del cuestionario y la confidencialidad y protección de datos de carácter personal. La recogida de datos se realizó a través de cuestionarios en papel y lápiz, y fueron analizados y custodiados conforme a las guías de la Declaración de Helsinki de la Asociación Médica Mundial (AMM) (64ª Asamblea General, Fortaleza, Brasil, octubre 2013).

\section{INSTRUMENTOS}

Escala de Ansiedad y Depresión Hospitalaria -HADS-57- (Zigmond y Snaith, 1983; adaptación al castellano de Rico, Restrepo y Molina, 2006). Es un autoinforme con 14 ítems puntuados de 0 a 3. Con esta escala se evalúa la sintomatología de ansiedad y depresión, con 7 ítems cada una: No síntomas (0-7 puntos); Caso dudoso (8-10 puntos); Caso probable (11-14); Muy probable (15-21). Ha sido validada en población oncológica obteniendo buenos parámetros de fiabilidad. En nuestro estudio solo se ha utilizado la dimensión de depresión, obteniéndose un índice de fiabilidad de de Cronbach de .86 . 
Escala de Resiliencia (Wagnild y Young, 1993; adaptación al castellano de Novella, 2002). Se compone de 25 ítems evaluados en escala tipo Likert de 1 a 7 puntos (1= Totalmente en desacuerdo; 7=Totalmente de acuerdo). La corrección se realiza mediante la suma de las puntuaciones de cada ítem, obteniendo: No resiliencia (25-74 puntos); Resiliencia baja (75-100 puntos); Resiliencia media (101-125 puntos); Resiliencia alta (126-150 puntos) y Resiliencia muy alta (151-175 puntos). Además de proporcionar una medida global de resiliencia aporta información sobre cinco subdimensiones: ecuanimidad, perseverancia, confianza en sí mismo, satisfacción personal y sentirse bien solo. En nuestra muestra, el índice de fiabilidad de de Cronbach de la dimensión global de resiliencia fue de .91 .

\section{RESULTADOS}

Para dar respuesta al primer objetivo, se realizó un análisis descriptivo de las variables del estudio tanto para la muestra total como para el grupo de mujeres supervivientes de cáncer y sin cáncer de manera diferenciada (Tabla 1).

Tabla 1. Estadísticos descriptivos de la muestra total, mujeres supervivientes de cáncer y sin cáncer

\begin{tabular}{llllllll}
\hline & & Total & & \multicolumn{2}{c}{$\begin{array}{c}\text { Mujeres } \\
\text { supervivientes }\end{array}$} & \multicolumn{2}{c}{$\begin{array}{c}\text { Mujeres sin } \\
\text { cáncer }\end{array}$} \\
\hline & Media & $\boldsymbol{D T}$ & Rango & Media & $\boldsymbol{D T}$ & Media & $\boldsymbol{D T}$ \\
\hline Edad & 44.27 & 11.79 & 44 & 52.20 & 8.85 & 38.78 & 10.41 \\
\hline Depresión & 3.75 & 3.47 & 16 & 4.11 & 3.98 & 3.49 & 3.07 \\
\hline Resiliencia & 138.90 & 19.82 & 97 & 142.86 & 13.85 & 136.15 & 22.76 \\
\hline
\end{tabular}

Como se observa, no se detectan síntomas de depresión patológicos ni en la muestra total, ni en los grupos de mujeres supervivientes y sanas, y toda la muestra se caracteriza por mostrar niveles altos de resiliencia. Sin embargo, las mujeres que enfrentaron esta enfermedad muestran mayores valores en depresión y resiliencia.

Para cubrir nuestro segundo objetivo y con el fin de estudiar si las diferencias entre los grupos de mujeres con y sin cáncer eran estadísticamente significativas, se llevó a cabo un análisis de varianza (ANOVA) de un factor. De los resultados se extrae que únicamente existieron diferencias estadísticamente significativas en la resiliencia, a favor de las mujeres que padecieron esta enfermedad $(F[1,109]=9.82, p<.01)$. Sin embargo no ocurrió lo mismo con la depresión ( $F[1,109]=$ 2.81, $p>05)$.

Para responder al tercer objetivo y con el fin de confirmar relaciones causales entre la depresión y la resiliencia, se realizó un análisis de regresión múltiple por pasos controlando el efecto de haber padecido o no cáncer y la edad de las mujeres. Se estableció la depresión como variable dependiente y la resiliencia como variable predictora. En el primer paso se introdujo las variables cáncer y edad, y en un segundo paso la resiliencia. Los resultados del análisis pueden observarse en la Tabla 2. 
Tabla 2. Análisis de regresión múltiple por pasos: predictores de la depresión

\begin{tabular}{lcccccc}
\hline & $R^{2}$ & Cambio en $R^{2}$ & $F$ & $\beta$ & $t$ & $p$ \\
\hline $\begin{array}{l}\text { VD: Depresión } \\
\text { Modelo 1 }\end{array}$ & .28 & .28 & $\mathbf{4 1 . 2 9 * * *}$ & & & \\
$\quad$ Resiliencia & & & & -.52 & $\mathbf{- 6 . 4 3 * * *}$ & .000 \\
Modelo 2 & .31 & .03 & $\mathbf{2 3 . 8 6 * * *}$ & & & \\
$\quad$ Resiliencia & & & & -.56 & $\mathbf{- 6 . 8 2 * * *}$ & .000 \\
$\quad$ Cáncer & & & & .18 & $\mathbf{2 . 2 2 *}$ & .02 \\
\hline$* p<.05$ & & & & & \\
$* * * p<.001$ & & & & &
\end{tabular}

En el modelo 1 la resiliencia explicó el $28 \%$ de la varianza de la depresión $\left(R^{2}=.28\right)$, mostrando capacidad predictiva negativa estadísticamente significativa $(\beta=-.52)$. En el modelo 2 , la capacidad explicativa se vio incrementada de manera significativa en un 3\% como consecuencia del efecto de sobrevivir al cáncer $\left(\mathrm{R}^{2}=.31\right)$, que mostró una relación positiva con la depresión $(\beta=.18)$, prediciendo mayores niveles en mujeres que pasaron esta enfermedad. Los resultados se confirmaron con independencia del efecto de la edad.

\section{DISCUSIÓN}

Los resultados obtenidos evidencian la ausencia de síntomas depresivos patológicos en la muestra total, como era de esperar dado que presentar un cuadro psicopatológico era uno de los criterios de exclusión del estudio. A pesar de ello, se encontraron niveles superiores de depresión en nuestra muestra de mujeres supervivientes de cáncer de mama, lo que se encuentra en consonancia con el estudio de Burgess et al. (2005), Mitchell et al., (2014) y Valderrama y Sánchez (2018) quienes observaron mayor tendencia a experimentar sentimientos negativos y secuelas emocionales tras la superación de la enfermedad.

Por otro lado, también son estas mujeres las que perciben mayores niveles de resiliencia de manera estadísticamente significativa (Acevedo y Ortiz, 2011; García et al., 2015; García-Serrablo et al., 2011). De acuerdo a Fernández y García (2012) la exposición al acontecimiento traumático de ser diagnosticada de cáncer facilita un proceso de crecimiento personal, asumiendo que la superación de la enfermedad conlleva fortalecimiento y una mayor capacidad para sobreponerse y manejar eficazmente preocupaciones y sentimientos negativos.

En cuanto a la relación entre la depresión y la resiliencia, los resultados arrojan una relación negativa entre ambas variables, sugiriendo menores niveles de depresión cuanto mayores niveles de resiliencia existan. Se establece así a la resiliencia como factor de protección predictor de menores niveles de estados depresivos (Hu et al., 2018; Markovitz et al., 2015).

A pesar de que padecer cáncer parece erigirse como factor de riesgo en la experimentación de síntomas depresivos (Acosta et al., 2017), el desarrollo de mayores niveles de resiliencia actuaría como un factor de protección clave que amortigua el efecto del cáncer sobre el desarrollo de sintomatología depresiva, resultados acordes a los encontrados por Cucarella (2013), Gotay et al. (2007) y Valderrama y Sánchez (2018).

Los análisis realizados permiten establecer las bases empíricas sobre las que desarrollar intervenciones que prevengan comorbilidades y el desarrollo de trastornos emocionales. De acuerdo a Viniegras y Blanco (2010), conocer la influencia que ejerce el perfil psicológico sobre la presencia 0 no de depresión permite su prevención y/o detección temprana, disminuyendo el coste de los tratamientos e incrementando el bienestar psicológico. 
El refuerzo de la capacidad para resistir y tolerar los obstáculos es esencial para fomentar un proceso de crecimiento personal ante situaciones adversas y crisis vitales, siendo especialmente relevante en población vulnerable a padecer trastornos emocionales, como son las supervivientes de cáncer de mama. Nuestros resultados sugieren que, la puesta en marcha de programas que refuercen la resiliencia en mujeres que han superado la enfermedad, permitiría igualar los niveles de depresión a mujeres sanas.

De cara a futuros estudios sería interesante realizar incluir las subdimensiones de resiliencia y realizar evaluaciones longitudinales que nos permitieran observar la evolución de los estados emocionales de las mujeres desde el momento del diagnóstico y durante el proceso de tratamiento adyuvante.

\section{CONCLUSIONES}

A la luz de los resultados obtenidos mediante el presente estudio se concluye que:

A pesar de no presentar síntomas de depresión patológicos, las mujeres supervivientes de cáncer de mama presentan niveles ligeramente superiores a las mujeres sin cáncer, si bien estas diferencias no son estadísticamente significativas.

Las mujeres supervivientes de cáncer de mama muestran mayores niveles de resiliencia de manera estadísticamente significativa.

La resiliencia es el principal factor que explica y predice negativa y estadísticamente significativa la sintomatología depresiva.

El cáncer actúa como factor de riesgo ante la depresión, con capacidad predictiva positiva estadísticamente significativa.

Cuando el cáncer se conjuga con el factor protector que ejerce la resiliencia, se contrarresta el impacto del cáncer en la manifestación de síntomas depresivos.

El diseño de programas que refuercen la resiliencia actuaría como intervención preventiva de la depresión tanto en población sana como en mujeres de oncología mamaria.

La inclusión de las subdimensiones de resiliencia permitiría una actuación más eficaz que prevenga comorbilidades psicopatológicas y alteraciones emocionales graves.

\section{REFERENCIAS BIBLIOGRÁFICAS}

Acosta Quiroz, C. 0., Tánori Quintana, J., García Flores, R., Echeverría Castro, S. B., Vales García, J. J., y Rubio Rubio, L. (2017). Soledad, depresión y calidad de vida en adultos mayores mexicanos. Revista Psicología y Salud, 27(2).

Baker, F., Denniston, M., Haffer, S. C., y Liberatos, P. (2009). Change in health related quality of life of newly diagnosed cancer patients, cancer survivors, and controls. Cancer, 115(13), 30243033.

Bellizzi, K. M., y Blank, T. 0. (2006). Predicting posttraumatic growth in breast cancer survivors. Health Psychology, 25(1), 47.

Burgess, C., Cornelius, V., Love, S., Graham, J., Richards, M. y Ramirez, A. (2005) Depression and anxiety in women with early breast cancer: five year observational cohort study. BMJ,330; $702-5$.

Consejería de Salud (s.f.). Registro del Cáncer Andaluz. Plan Integral de Oncología de Andalucía 2002-2006.

Cucarella, M. S. P. (2013). Bienestar emocional, imagen corporal, autoestima y sexualidad en mujeres con cáncer de mama (Doctoral dissertation, Universitat de València, Departamento de Personalidad, Evaluación y Tratamientos Psicológicos). 
Fernández, L. C., y García, M. L. R. (2012). El poder de la resiliencia generado por el cáncer de mama en mujeres en Puerto Rico. Revista puertorriqueña de Psicología, 23(1), 109-126.

García-Herrera, J., Nogueras, E., Muñoz, F., y Morales, J. (2011). Guía de Práctica Clínica para el tratamiento de la depresión en Atención Primaria. Málaga: Distrito Sanitario Málaga Ugc Salud Mental Hospital Regional Universitario «Carlos Haya.

García-Maroto Fernández, S., López Delgado, M. L., y Latorre Postigo, J. M. (2015). Ansiedad, resiliencia e inteligencia emocional percibida en un grupo de mujeres con cáncer de mama. Ansiedad y estrés, 21.

García-Serrablo, P., Acevedo, S., y Ortiz, N. (2011). Benefit finding in breast cancer patients: The perspective of Puerto Rican women. In 118th APA Convention.

Gotay, C. C., Ransom, S., y Pagano, I. S. (2007). Quality of life in survivors of multiple primary cancers compared with cancer survivor controls. Cancer, 110(9), 2101-2109.

Guil, R., Zayas, A., Gil-Olarte, P., Guerrero, C., González, S., y Mestre, J. M. (2016). Bienestar psicológico, optimismo y resiliencia en mujeres con cáncer de mama. Psicooncología, 13(1), 127.

Hu, T., Xiao, J., Peng, J., Kuang, X., y He, B. (2018). Relationship between resilience, social support as well as anxiety/depression of lung cancer patients: A cross-sectional observation study. Journal of cancer research and therapeutics, 14(1), 72.

Krebber, A. M. H., Buffart, L. M., Kleijn, G., Riepma, I. C., De Bree, R., Leemans, C. R., ... y Verdonck de Leeuw, I. M. (2014). Prevalence of depression in cancer patients: a meta analysis of diagnostic interviews and self report instruments. Psycho Oncology, 23(2), 121-130.

Markovitz, S. E., Schrooten, W., Arntz, A., y Peters, M. L. (2015). Resilience as a predictor for emotional response to the diagnosis and surgery in breast cancer patients. Psycho Oncology, 24(12), 1639-1645.

Mitchell, A. J., Ferguson, D. W., Gill, J., Paul, J., y Symonds, P. (2014). Depression and anxiety in long-term cancer survivors compared with spouses and healthy controls: a systematic review and meta-analysis. The lancet oncology, 14(8), 721-732.

Molano, N. J., y Varela, P. E. V. (2017). Percepción acerca del cáncer de mama en un grupo de mujeres de un hospital en Popayán, Colombia Breast cancer perception in a group of women in a hospital in Popayán, Colombia. Revista MHSalud, 13(2).

Montiel Castillo, V. E., Álvarez Reyes, 0. L., Guerra Morales, V. M., Herrera Jiménez, L. F., y Fernández Castillo, E. (2016). Síntomas depresivos en mujeres con cáncer de mama en intervalo libre de enfermedad. Medisan, 20(02), 184-191.

Newport, D. J., y Nemeroff, C. B. (1998). Assessment and treatment of depression in the cancer patient. Journal of psychosomatic research, 45(3), 215-237.

Novella, A. (2002). Incremento de la resiliencia luego de la aplicación de un programa de psicoterapia breve en madres adolescentes. Tesis para optar el grado de Magíster en Psicología, mención en Psicología clínica y de la salud.

Organización Mundial de la Salud (2004). La relación entre la salud física y la salud mental. Trastornos concurrentes. Día Mundial de la Salud Mental. Génova: Organización Mundial de la Salud.

Rico, J.L., Restrepo, M. y Molina, M. (2006). Adaptación y validación de la escala hospitalaria de ansiedad y depresión (HAD) en una muestra de pacientes con cáncer del instituto nacional de Cancerología de Colombia. Avances en Medición, 3, 73-86.

Rodríguez, V. P., Amboage, A. M., Blázquez, M. H., Torres, M. Á. G., y Gaviria, M. (2015). Depresión y cáncer: una revisión orientada a la práctica clínica. Revista Colombiana de Cancerología, 19(3), 166-172. 
Sociedad Española de Oncología Médica (2017). Las cifras del cáncer en España.

Sociedad Española de Oncología Médica (2018). Las cifras del cáncer en España.

Valderrama Rios, M. C., y Sánchez Pedraza, R. (2018). Trastornos de ansiedad y depresión en relación con la calidad de vida de pacientes con cáncer de mama en estadio localmente avanzado 0 diseminado. Revista Colombiana de Psiquiatría, 47(4), 211-220.

Vázquez, 0. G., Benjet, C., Juárez García, F., Castillo, E. R., Rosas, A. R., Ponce, J. L. A., ... y Aguilar, S. A. (2015). Propiedades psicométricas de la Escala Hospitalaria de Ansiedad y Depresión (HADS) en una población de pacientes oncológicos mexicanos. Salud mental, 38(4), 253-258.

Viniegras, C. R. V. G., y Blanco, M. G. (2010). Bienestar psicológico y cáncer de mama. Avances en psicología latinoamericana, 25(1), 72-80.

Wagnild, G., y Young, H. (1993). Development and psychometric. Journal of nursing measurement, 1(2), 165-17847.

Zigmond, A. S., y Snaith, R. P. (1983). The hospital anxiety and depression scale. Acta psychiatrica scandinavica, 67(6), 361-370. 
